\title{
Assessing the potential use of blockchain technology to improve the sharing of public health data in a western Canadian province
}

\author{
Sarah Murphy ${ }^{1} \cdot$ Paul Reilly $^{2} \cdot$ Teresa Murphy $^{1}$
}

Received: 2 October 2020 / Accepted: 4 March 2021 / Published online: 16 March 2021

(c) IUPESM and Springer-Verlag GmbH Germany, part of Springer Nature 2021

\begin{abstract}
This exploratory, qualitative study set out to identify the encountered and perceived barriers to public health (PH) data sharing in a Canadian province with a view to assessing blockchain technology as a potential solution. A topic guide was developed, based on previous research in the area. This was then utilised for ten in-depth, semi-structured interviews with PH professionals between 27 May and 18 June 2019. Each stage of research was congruent with the philosophical underpinning of Gadamerian hermeneutic phenomenology. The major themes that emerged from the data collected were related to the information systems in use, data quality and ownership, as well as client identity management. The recurring core theme throughout all interviews was related to ineffective leadership and management, contributing to each major theme. Overwhelmingly the results show that the majority of barriers faced in this province are human-related. It is concluded that while blockchain technology shows promise for enhancing data sharing in healthcare, it is still many years away from being implemented in this Canadian province. As the results of this study indicate, there are human related barriers that could be addressed in the meantime, which are outside the scope of a technical solution. Future work should explore the perspectives of other stakeholders, such as the provincial government to fully understand the potential for using blockchain to share PH data in this province.
\end{abstract}

Keywords Public Health $\cdot$ Health Informatics $\cdot$ Health Information Systems $\cdot$ Data Sharing $\cdot$ Blockchain

\section{Introduction}

The increasing complexity of the healthcare ecosystem has inevitably led to a number of data privacy and security challenges for key stakeholders in countries like Canada. While the majority of medical records have been digitised, they are often stored in silos dispersed across medical facilities around the globe. This has implications for public health $(\mathrm{PH})$ intelligence, which relies on the sharing of complete and accurate information between organisations. Blockchain technology is being touted as having the potential to address

Sarah Murphy

marahsurphy@gmail.com

Paul Reilly

p.j.reilly@sheffield.ac.uk

Teresa Murphy

teresaageary@gmail.com

1 Public Health Informatics, Western Canada, Canada

2 University of Sheffield, Sheffield S10 2TN, UK these issues of information sharing within the healthcare sector. This paper sets out to identify the encountered and perceived barriers to data sharing in $\mathrm{PH}$ within a western Canadian province through a qualitative analysis of ten semi-structured interviews with a range of healthcare professionals between 27 May and 18 June 2019. The aim of this study was to first understand the barriers to data sharing in this jurisdiction, in order to investigate whether blockchain technology could be used to enhance the data sharing of PH information provincially and subsequently nationally.

\section{Current state of data sharing in public health}

Health data sharing is essential at an individual level for patient care, and at a broader level for population health and outbreak management. It is a complex task to achieve, as all healthcare stakeholders including patients, clinicians, pharmacists, biomedical laboratories, national and international PH agencies depend on it. Data sharing is further 
complicated by the fact that valuable $\mathrm{PH}$ information may be generated from many different sources. These range from disease surveillance, family health, biostatistics and informatics, communicable disease prevention and immunisation programmes [1]. Pertinent data is also stored in the form of electronic medical records (EMRs) held by hospitals, $\mathrm{PH}$ units and general practitioners. This data is acquired, stored and accessed in a variety of formats, which makes it difficult to create and maintain an accurate, comprehensive and accessible database of PH information. Nevertheless, the consensus amongst researchers is that $\mathrm{PH}$ agencies need timely access to such data in order to effectively plan and operate services to improve population health $[2,3]$. The response to the 2003 severe acute respiratory syndrome (SARS) epidemic displayed the power of real-time data sharing in supporting swift PH responses [4]. More recent health emergencies, such as the 2014 Ebola epidemic and the ongoing COVID-19 pandemic, further highlight the importance of rapid data sharing beyond geographical boundaries. The availability of accurate data is critical for epidemiological modelling, prioritisation of surveillance, as well as understanding infection risk factors and transmissibility of disease at all levels of PH management [5, 6]. At the local level, PH data supports targeted interventions, at the national level the data informs federal planning and policy making, and globally the data provides essential metrics for managing international health crises [7]. The lack of interoperability between each level remains an issue for $\mathrm{PH}$, as the systems are often disjointed providing inadequate communications, leading to costly workarounds. For example, in an effort to address these issues a PH clinical and surveillance system implemented in Canada, at a cost of 115 million CAD, went 420 percent over budget, has an estimated annual cost of 14 million CAD and ultimately failed to achieve implementation in all jurisdictions as intended [8]. Similar issues exist elsewhere, in relation to the number of systems created within the same jurisdiction. Due to years of single purpose, disease specific systems created at the US Centre for Disease Control and Prevention (CDC), there are reportedly now more than 110 surveillance systems in existence [9]. The call for greater data exchange capabilities between $\mathrm{PH}$ information systems provides an undeniable business need for a better solution [10, 11]. PH data is also valuable for secondary uses, such as research and the development of technology. The advantages of improved data sharing and availability are well documented, and include the development of more effective $\mathrm{PH}$ programmes, reproducible and consistent research and cost efficiencies [12-14]. The barriers to the sharing of health data in general, are also well known. A recent systematic review by van Panhuis et al. identified twenty barriers to international PH data sharing, such as restrictive data format, protection of privacy, language barriers and lack of resources and guidelines [7].
This paper sets out to build on this review, which focused at the global level, by exploring the specific perceived barriers encountered within a more narrowly defined jurisdiction in order to understand how recent technologies, namely blockchain, might address the issues identified. It has been reported that blockchain technology can enhance health data interoperability standards while maintaining the required security and privacy for the management of such data [15].

\section{Blockchain technology in healthcare}

A blockchain is a distributed ledger, or database, of transactions which are immutable and traceable [16]. There are three main blockchain designs, public-which is not controlled by any single entity and is open source, allowing any actor to participate without restriction, private-which is permission based and accessed only by authorised actors, and lastly hybrid - which provides flexibility and the ability to designate subsets of data to be available publicly or privately [17]. There have been few studies exploring blockchains potential application within the healthcare sector to date, particularly for PH data. According to the Gartner Hype Cycle, which interprets evolving technology hype, blockchain technology has moved from the 'Peak of Inflated Expectations' where there were some success stories but also many failures, to the 'Trough of Disillusionment'. This phase typically sees a sharp decline in interest when there is a failure to deliver on the implementations to date, and where mass adoption is estimated to be five to ten years away as of 2019 [18]. Thus far, much of the hype over blockchain has surrounded its application in the financial sector. The first blockchain supported a cryptocurrency named Bitcoin, which was introduced in 2008 by Satoshi Nakamoto [19]. A detailed technical explanation is beyond the remit of this paper, but it is worth noting that it was conceived as a decentralised digital cash system, independent of a central third-party validating transactions, that employs cryptography and a shared, distributed ledger [19]. This concept effectively removes intermediaries by creating a system where two strangers can transfer value to each other, without prior established trust in a secure, indisputable way [16]. The research indicates that this technology could be applied to healthcare data as an enhanced method of protection, also supporting interoperability of existing systems [20]. The literature suggests that blockchain is an appropriate solution in those situations where there are several stakeholders, trust is lacking, and reliable and accountable tracking is required [21]. PH data certainly qualifies as an area requiring further research around blockchain technologies. Blockchain technology is a complementary solution that can be combined with existing processes to enhance them. In the context of healthcare, this technology has already been successfully deployed in certain locations. Recent systematic 
literature reviews by Engelhardt (2017), and Agbo, Mahmoud and Eklund (2019) regarding the use of this technology in healthcare found that enhancing data sharing, EMR management and access control are at the forefront [22, 23]. Estonia, for example, was the first country to implement blockchain technology at a national level, with 'Guardtime' being used to manage the EMRs for over 1 million citizens [22, 24, 25]. The healthcare records of each citizen are protected by blockchain technology, affording them the right to grant or refuse permission for their data to be accessed and used by third parties [26]. The CDC in America has also begun to explore how distributed ledgers can help PH practitioners respond more quickly to crises, inviting proposals for how blockchain could be integrated into healthcare in the US [27, 28]. Yet, to date there has been little research exploring the potential barriers to the implementation of blockchain within the healthcare sector. Some research does suggest that the challenges to implementation relate to scalability, cyberattacks and high energy consumption $[29,30]$. It has also been recognised that while blockchain technology can reduce cost in the longer term, the initial outlay for implementation is significant [31]. This paper sets out to build on this research by exploring how PH data might be shared using these technologies within a Canadian province and whether it is a feasible solution. In Canada, there are thirteen individual health care insurance plans, with each province and territory being responsible for the management and delivery of healthcare services to their residents [32]. Within the province under investigation, there are a number of health regions (HRs) both provincial and regional, each with a further level of autonomy regarding the management and delivery of health care including PH. Due to the limited amount of information available specific to this Canadian province, and the exploratory nature of the research, a qualitative research design has been chosen. This research was guided by the philosophical hermeneutics of Gadamer, which seeks to expand understanding through dialogue between people, and between researchers and text [33]. Through qualitative semi-structured interviews, the perspectives of PH professionals were obtained with the goal of identifying barriers to sharing PH data in the province. A phenomenological design was adopted as it allows the researcher to explore the essence of a particular phenomenon through the narratives of individuals with relevant experience and knowledge regarding the phenomenon [34,35]. The interviews drew on the experiences and knowledge of the participants, based on their involvement in the industry. For this reason, hermeneutical phenomenology, which focusses on the subjective experience of the individual, was the chosen research methodology [36]. The research was undertaken with an inductive approach and an interpretive understanding of the findings. The questions within the study elicited the participants' individual perspectives and beliefs regarding barriers to data sharing within the $\mathrm{PH}$ system, and the impact of this. This flexible, qualitative research design aims to provide an answer to the following research question:

What are the barriers to data sharing and can blockchain technology enhance data exchange in the context of $\mathrm{PH}$ in a Canadian province?

\section{Sample}

A purposive, non-probability sampling strategy was employed in order to identify PH professionals with relevant roles in both the sector and jurisdiction under analysis. The ten participants had between five and thirty years of experience in the sector, eight of whom were employed by the provincial Public Health Organisation (PHO), one by a $\mathrm{HR}$ and one by a neighbouring territory. It was considered important to include both the perspectives of the provincial organisation and their typical partners in PH data sharing. A number of the interviewees could also speak about $\mathrm{PH}$ with reference to their experience holding multiple roles, such as Participant A, who had worked as a PH informatics manager and a registered nurse (see Table 1).

In-depth, semi-structured interviews were the chosen method of data collection. Data were collected between 27 May and 18 June 2019. Interviews were conducted

Table 1 Participants' roles

\begin{tabular}{ll}
\hline Participant & Role(s) \\
\hline A & Public Health Informatics Manager; Registered Nurse \\
B & Public Health Informatics Lead; Registered Nurse \\
C & Public Health Privacy Officer \\
D & Communicable Disease Analyst \\
E & Director of Health Informatics Projects \\
F & Project Manager of Health Informatics Projects \\
G & Regional Public Health Informatics Specialist; Registered Nurse \\
H & Public Health Informatics Director; Registered Nurse \\
I & Territorial Public Health Informatics Manager; Registered Nurse \\
J & Public Health Medical Director; Medical Doctor \\
\hline
\end{tabular}


face-to-face with eight of the participants in the boardroom of the PHO. One interview had to be suspended halfway through due to a PH emergency, but was completed a few days later. The remaining two interviews were conducted via teleconference, due to participant location. Open-ended questions were used to allow for individual interpretation and for participants to direct the conversation based on their own experiences. A topic guide was followed to ensure a level of consistency and structure across interviews, while also affording a level of flexibility for the discussion to naturally evolve. To ensure the scope of the interviews was satisfactory, the topics discussed were informed by previous research in the area; the interview schedule was also piloted in May 2019 in order to ensure the questions were easily understood. Gadamer believed that every individual has a unique perspective, or view of the horizon, based on their own life experiences and knowledge [37]. The information gathered in each interview added to the researcher's horizon and was brought into the subsequent interview, deepening the circle of understanding with each interaction. In line with previous work [38], no further participants were sought once data saturation was reached.

\section{Gadamerian hermeneutic phenomenology and the role of the researcher in data collection}

For a Gadamerian hermeneutic phenomenological study, it is critical to acknowledge but not bracket the assumptions, biases and prejudices of the researcher relating to the phenomenon under investigation [37]. Rather than eradicate, or bracket researcher prejudice, the aim was to discuss the perceived and encountered barriers to data sharing in $\mathrm{PH}$; thus, creating a 'fusion of horizons' about this phenomenon throughout the interview process. Data was collected by the lead researcher, a public health informatics professional with insider status due to their employment within the same organisation as the majority of participants. This increased the level of trust and confidence shared in the researcher/ participant relationship, allowing for rapport to be easily built. This also meant that the language and jargon used by participants were immediately understood, allowing for efficient data collection and the ability to clarify any points after the fact, as required [39].

\section{Ethics}

Ethical approval was granted by the host institution prior to data collection beginning, in October 2018. The lead researcher also sought permission from the primary research site before key stakeholders were approached to participate in the study. Each interviewee was provided with an information sheet outlining the objectives of the study and their right to withdraw at any point. In order to respect their anonymity and confidentiality, their respective organisations and specific locations are not identified. All interviews were recorded and transcribed prior to analysis.

\section{Data analysis}

Gadamer's hermeneutics dictated the approach to data analysis. A qualitative thematic approach was used to analyse the data [40]. Once the interviews and transcription were completed, the next step was to listen to the audio recording for each interview repeatedly. This allowed the researcher to become extremely familiar with the participants' experiences and perspectives prior to the data analysis. Transcripts were then printed and subjected to a manual, iterative coding process, which involved noting common phrases and words used by the interviewees and devising codes in a systematic way [38].Once initial themes were identified, the transcripts were reviewed once more, with further common words and phrases identified and added to the documented barriers. Through this interaction with, and interrogation of the text, data analysis continued. This method aligned with Gadamer's hermeneutic circle, where the researcher moves from the whole to the parts, and back to the whole again [37, 41].

\section{Limitations}

As is typical for a qualitative research project, the results were derived from the researcher's analysis, which was by definition interpretive. However, this was congruent with the Gadamerian hermeneutic phenomenology research design which does not bracket one's assumptions and pre-judgements from the data analysis.

\section{Results}

\subsection{Barriers to data sharing within the provincial PH system}

All of the participants reported that data were collected for $\mathrm{PH}$ functions such as disease surveillance, clinical documentation, programme evaluation and planning, as well as identifying outbreaks. Nevertheless, there was a multitude of barriers and challenges described by each participant, informed by both their role and direct experience working in the $\mathrm{PH}$ sector. Having analysed the data generated during the interviews, the following themes and subthemes emerged (see Table 2). 
Table 2 Perceived barriers to public health data sharing

\begin{tabular}{|c|c|}
\hline Major Theme & Subtheme (n) \\
\hline \multirow[t]{4}{*}{ Legislative } & Information Systems in Use (10) \\
\hline & Mandate on Information Sharing (8) \\
\hline & Interpretation of Privacy Legislation (6) \\
\hline & Privacy Breaches (4) \\
\hline Technical & Data Integrity (6) \\
\hline Cultural & Data Territorialism (7) \\
\hline Social & Client identity management (4) \\
\hline Political & Policies that limit access to government datasets (6) \\
\hline
\end{tabular}

\subsection{Information systems in use}

The majority of the HRs in the province document and store PH data in their own, siloed information systems with limited, or no integration with the Public Health System (PHS). All of the interviewees argued that the PHS had thus far failed to provide a cohesive provincial system due to factors such as inadequate system design, lack of stakeholder engagement early in the project and poor direction and leadership which resulted in multiple $\mathrm{PH}$ information systems across the HRs. The Public Health Informatics Lead reflected on this barrier, "where I think it started to go off the rails is the [PHS] itself was initially conceived by the surveillance team" (Participant B). Surveillance personnel typically access the data from the database directly, and were said to be less concerned with the front-end design, which meant that it didn't meet the medical legal requirements for clinical documentation: "they hit the nail on the head from a surveillance perspective, but missed the boat on it being a really effective and slick front-end clinical system, both in terms of ease of use, but also it doesn't meet all the medical legal requirements for clinical documentation. For example, you can delete information out of the system and when you're documenting medical legal information, that's the equivalent of tearing a page out of a paper file" (Participant B).

It was suggested that this was one of the main reasons that the majority of HRs refuse to use the PHS directly. Compounding this issue, Participant B confirmed that the largest $\mathrm{HR}$, responsible for 40 percent of the province's population, had exercised their right to move away from using the PHS in June 2019 and deployed another separate PH information system. This point was echoed by the Public Health Medical Director (Participant J) who noted that "historically, there hasn't been a mandate or a requirement for all health regions to use the same information system". This theme emerged from all the interviews and was widely viewed as a root cause of many of the problems related to sharing $\mathrm{PH}$ data in the province. The Public Health Informatics Manager stated that "the majority of the work done in public health is provincially driven and there's provincial outcomes that need to be given to the minister of health; I think one of the big things is bring it back under the same provincial umbrella" (Participant A). Two of the participants, both registered nurses, reflected on the huge variation across the HRs but nevertheless felt that there was no rationale for using a variety of information systems across the province: "you don't have to implement programmes the same way because each community is different. And I get that but to have people on the same system would help" (Participant H). The Communicable Disease Analyst, who worked with all the HRs in their role at the PHO, expressed their frustration at the lack of cohesion between the regions: "it's always going to be difficult when we have battling health regions that each want to go their own way and aren't putting in the full effort to get the data into the required system" (Participant D).

All participants described the disjointed PH system in the province as a major barrier to data sharing. The Public Health Informatics Manager detailed how the delivery of PH programmes had previously been provincially controlled until the provincial government had transferred this management function to the numerous HRs, who did not have concurrent access to, or use of the same $\mathrm{PH}$ information system. The Public Health Medical Director also articulated this frustration: "it's a detriment for the public health programme and it does allow [the HRs] to not share specific variables with us. Even if those variables might be in their information system, they simply haven't come over [to the PHS] and it's been very difficult to understand, are those variables even collected? If they're collected, are they entered?" (Participant J).

\subsection{Mandate on information sharing}

Eight of the interviewees believed that the provincial government should provide greater clarity in terms of the scope of PH information sharing. The Public Health Informatics Manager emphasised the need for the provincial government to enforce standards: "I think there needs to be the enforcement on what information needs to go in and how. I think that's where in [this province] in particular we're really lacking. If a health region chooses not to follow that, sure we can escalate to the provincial government, but sometimes it falls on deaf ears" (Participant A). Participants perceived that some of the HRs resisted sharing data with the province and wanted to retain it within their silos. For example, the Public Health Informatics Lead felt that patient interests should be paramount: "the news flash is, everybody's paid by the provincial government. So, buck up, shut up and do what's in the best interest of the patient" (Participant B). One participant who had previously worked in other Canadian provinces, and was familiar with the PH system in the United States, argued that they had never experienced anything like this 
'tribal carve-up': “it's never been the position in [other provinces] public health. You know, it was a much more collaborative public health process. I think there's more of a sense that we're all in the same mission. I don't mind going on record but that's not the perspective in this province. It's very tribal. And that's too bad because it makes it harder to do work, there's enough work for everyone without having to deal with these kinds of extra issues" (Participant J). All were enthusiastic about two mandates recently introduced by the provincial government to ensure the PHS was updated regularly with relevant data pertaining to immunisation records and reportable communicable diseases (RCD) for the provincial population. Some of the more experienced interviewees had a 'wait and see' attitude to these new mandates: "Each health region has signed letters of understanding for these mandates. Now at least there is a formal mechanism to leverage that should someone be so bold as to have the kahunas to do it. So, when there is resistance, I'm hoping that they won't, just bend in the breeze under the pressure because there will be pressure" (Participant B).

\subsection{Interpretation of privacy legislation}

Most participants identified varying interpretations of privacy legislation across the HRs as a major barrier to $\mathrm{PH}$ data sharing in the province. While legislation regarding PH data was considered amongst the most robust in Canada, the Public Health Privacy Officer conceded that "some areas will interpret more conservatively than other areas with respect to their obligations" (Participant C). The Director of Health Informatics Projects provided further insight into this issue based on the implementation of a portal which enabled the public to view their laboratory results. Efforts to extend this portal to other HRs were initially hampered by the interpretations of 'conservative people' and this interviewee was frustrated by the delay and the waste of taxpayer money: "if I go back to how much did the taxpayers pay to actually have one health region basically accept the findings and realism, from the experience of another health region-way too much time." (Participant E). This participant felt strongly that HRs should be working in unison and should trust the due diligence done by each other when implementing these systems. Similarly, the Public Health Informatics Director criticised the "very complex roles and permission sets that were required at the beginning of this journey of the public health system" (Participant $\mathrm{H}$ ). They felt strongly that the professional judgement of $\mathrm{PH}$ staff should be given greater weight, rather than implementing solutions which actually turned out to be barriers to accessing information systems and ultimately PH data sharing.

\subsection{Privacy breaches}

Prior privacy breaches were cited by four of the participants as the reason why there was limited data sharing between HRs. The Territorial Public Health Informatics Manager argued that lawsuits, particularly relating to the release of patient data without their consent, meant that staff were often cautious about developments such as PHS: "It's people's experience. I tend to be a little bit more on the secure side only because I've lived in [two provinces] when they both had the lawsuits" (Participant I). The Public Health Informatics Manager spoke of the difficulties in managing inappropriate access to clinical data within these systems, often not knowing whether there was a legitimate reason for client records being examined by health professionals. The Public Health Informatics Lead provided examples of serious data breaches they witnessed while working as a frontline nurse, such as "an entire therapeutic abortion list coming across our fax machine in a unit where that should not have come" (Participant B). Hence, there were policies and training in place for $\mathrm{PH}$ staff to assist with proper data management. However, this was said to be only effective for users of the PHS and for data in electronic format. Some data is collected on paper forms which allow a variety of responses which are inconsistent and often times incomplete. Moreover, not all participants believed these breaches were a new phenomenon linked to the development of sophisticated information systems; the Public Health Informatics Manager asserted that "there was breaches probably way more often with paper. It just doesn't have quite the same impact" (Participant A).

\subsection{Data integrity}

Participants described the difficulty in accurately mapping data from numerous systems into one provincial system. The data is entered into the PHS manually by PH staff or by bulk uploads, data feeds or interfaces from other PH information systems with the goal of one comprehensive provincial database of PH information. For those that did not use PHS as their clinical documentation system, the required data was often sent via fax machine to the province or not submitted at all. The Communicable Disease Analyst acknowledged the gaps in $\mathrm{PH}$ data as a result of these variations: "if we're looking at our surveillance data for the past year, we're finding gaps in how it's been entered" (Participant D). The quality of the PH data was a great concern for the majority of the interviewees. A variety of methods were employed to ensure data quality within the PHS. Proactive approaches included the configuration of the information system to include mandatory fields for information gathering. However, this can only be applied to the HRs that directly used the PHS system and there was no mechanism 
to reject data submitted in paper format or via fax, which meant extra work for both the province and the HRs. Other methods included technical and business conformance standards such as specific messaging format: "the latest and the greatest on the market right now for health is FHIR, it's Fast Healthcare Interoperability Resources" (Participant F). Due to the fact PHS had recently been mandated by the provincial government as both the provincial immunisation and $\mathrm{RCD}$ repository, participants reported there was now the ability to enforce requirements on those data senders. The HRs sending required information to the province had to build their data feeds to these specifications, with specific code set values. According to the Project Manager of Health Informatics Projects: "the code set values that we've tried to use are the ones that we've discussed nationally, to make sure that if we ever want to be interoperable with other provinces, let's all talk the same language and the same code sets" (Participant F). The Public Health Informatics Director confirmed that the intention was to put the onus on HRs to maintain data quality and completeness, with data not meeting these standards rejected: "our fundamental principle is that data is fixed at source. If there's an issue on translation through our adapters and things like that, we have already started hiring a team that will fix the data" (Participant $\mathrm{H}$ ). Reactive methods included regular audits to identify issues with the existing data in PHS. In those cases, individual users or teams managing data feeds were often contacted to correct the data. The Director of Health Informatics Projects emphasised the importance of ensuring data integrity from these feeds; "At the end of the day you have to have good data. Crappy data leads to crappy decision making. So, having those proactive processes to go and actually make sure that data is as accurate as possible is absolutely critical." (Participant E). Cross-referencing the data in PHS with other sources was already said to have ensured more accurate $\mathrm{PH}$ data: "for example, if for meningococcal disease, what's been reported into [PHS] is different than what the laboratory has as a serogroup. Then we would follow up with the Public Health Unit and say, look, this was ' $w$ ' in the lab system, you've got it recorded as a 'y'." (Participant J). Obvious discrepancies were easily identified in some cases, such as "an immunisation that was supposed to be administered by intramuscular injection but had been recorded as being given orally" (Participant I).

\subsection{Data territorialism}

Those HRs that did not directly use PHS were said to be 'territorial' about the data in their individual systems. The majority of participants raised this as a major barrier to data sharing in the province. Most notably, the Public Health Informatics Manager asserted: “maybe it's they don't want to share because they don't want us to see what kind of work they are doing in their health region, or they don't feel that it's secure. If there's that level of ownership, then they don't want to share" (Participant A). The Project Manager of Health Informatics Projects provided some insight into this based on their experience creating the provincial immunisation repository. Each HR had a degree of autonomy in how they delivered their PH programmes, and one HR had a different schedule for childhood immunisations to the others, which meant that some of its records appeared invalid in the PHS and valid in the HR's system. The Public Health Privacy Officer had also experienced this sense of data ownership amongst the HRs: "you do see is a fair amount of data territorialism. We have (a number of) different health regions with a fair amount of autonomy. That says, I own this section of data, I own that section of data." (Participant C). The Public Health Privacy Officer provided further insight into the 'lack of control' felt by some of those HRs sharing data: "once you share data out that's your weakest link. You no longer, in a lot of ways, have control over that data. So, people naturally have concern around that" (Participant C). Overall, most participants felt that 'data territorialism' made no sense given that the provincial government was ultimately funding the activities of all HRs.

\subsection{Client identity management}

During the in-depth interviews the topic of client identity management was raised by four of the participants, all of whom have worked as frontline PH nurses in the past. This barrier was reported as an evolution of some of the care that was provided in the 1980s. Patients were presenting with an unknown illness that did not yet have a name, now known as HIV and AIDS “We just knew that people were getting really ill and we knew that it was in a segment of our population that were primarily gay men and that there was a lot of stigma associated with that" (Participant B). As a result of the associated social stigma, patients used pseudonyms which propelled this $\mathrm{PH}$ intensity to being anonymous “... trying to hide your identity because there were such significant consequences if you were inadvertently identified as someone that had essentially a fatal disease at that time. You couldn't get insurance and you could lose your job. And, you know, there were all sorts of ramifications and we've come a long way, but it's a bit of a cling on." (Participant B). This led to a lot of anxiety about having this sensitive information in clinical systems. Patients began to avoid providing their real identifiers. This was accepted by the $\mathrm{PH}$ care system as testing and treatment were prioritised; "At the time there was no process in place within public health to provide any kind of anonymity or cloaked identity. So, people made it up. They just kind of did their own thing, we were working in isolated databases at the time, so everybody kind of knew the rules by which they were playing within their own 
sandbox. But now that we're amalgamating this data and it's all coming into the same repository that creates incredible challenges." (Participant B). It was reported that PH has evolved with lax client identity rules as result of this and not because they were ever formalized anywhere. This creates complex challenges today, as described by the Public Health Informatics Lead "what record does this go on and how do you ensure that the person comes back and has continuity within their record when you're not validating that they are who they are. This issue leads to fragmented health records in some cases and in others can lead to incorrect information being recorded on the wrong clinical record" (Participant B). The Public Health Informatics Manager felt that "there's just this like cultural issue beyond public health where there is this fear of people finding out my business" (Participant A).When working as a PH nurse in a youth clinic, this participant also experienced concerns from the youths, an example was given as 'they didn't want to provide their care card number because they didn't want their parents to find out that they were using the services. Likewise, they don't want it in a provincial computer system. Because what happens if my friend's mom is a public health nurse, she's going to go look up my information and she's going to go tell my mom" (Participant A). The impact of these client identity issues is still present today. The Director of Health Informatics Projects said "the basic fundamentals of who is the patient, we have a hard time actually reconciling that. So, we built, unfortunately with taxpayers' money, a reconciliation process" (Participant E). This has proved a very costly and time-consuming issue, the repercussions of which are still emerging today.

\subsection{Policies that limit access to required datasets}

Another major barrier described by participants was the lack of access to and integration with other government data sets. PH relates to population health, and in order to have a clear picture of the health of the population and accurately plan programmes, participants felt that access to denominators was critical. Lack of active integration with the provincial client registry (PCR) was cited as a challenge for data sharing by six of the participants. The PCR is a provincial government patient database and every patient that receives any healthcare services in the province has a record in this system. The data consists of demographic information, including a unique identifier and is the source of truth for this information provincially. Participants felt that active integration with this database would alleviate some of the client identity issues described above. The Director of Health Informatics Projects suggested that this issue could have been avoided with effective leadership many years ago "again, the executive leadership perspective, 15 years ago someone in that role should have said-you want to play in our market. You will be connected to PCR in a matter of two years. And if not, we want to see the decommissioning plan for your system" (Participant E). If integration with PCR had been enforced, the foundation behind interoperability would be taken care of because issues of mismatching data would be minimised and access to the unique identifiers would be readily available for verification.

\subsection{Mixed views on future of blockchain in $\mathrm{PH}$ sector}

Four participants had never heard of 'blockchain', and therefore were unsure how it could be used to enhance data management and sharing in healthcare. "I am not familiar with blockchain, I've never heard of that term" (Participant $\mathrm{G})$. However, the majority were familiar with the concept and felt that the technology might be useful in the areas of data privacy, security and auditing. The Public Health Privacy Officer expressed enthusiasm for blockchain but acknowledged that they did not fully understand it: There is talk about it in the sense of being a privacy positive way of, of sharing information. I would say fundamentally, uh, despite having gone to a number of sessions that have tried to explain it and its involvement within different areas that involves sensitive information, banking, healthcare etc. I still do not really understand it" (Participant C).

The Director of Health Informatics Projects shared the same feeling "I'm familiar with the high level 50,000-foot concept behind it. Um, I have no understanding of how it could be applicable" (Participant E). Two participants were more concerned with the current state of information management and felt that efforts should be focussed on addressing these issues before looking at blockchain and other technological solutions “....in Canada we're still behind about actually getting to the point of being able to share data. The method of sharing data. That's another question." (Participant C). The Director of Provincial Projects concurred “... it kind of scares me just in the sense of we've got this thing called the fax machine that we have to get rid of. So, you know, I'm torn between the let's go, let's go take us out of the $70 \mathrm{~s}$ to even the two thousand's, um, and while it's a fantastic opportunity, I'm told, but, you know, where does it fit in?" (Participant E).

\section{Discussion}

The results of this study were broadly in alignment with the technical, economic and legal barriers identified in previous research into data sharing within the healthcare sector [7]. However, this paper also identified cultural barriers that might impede the integration of blockchain into $\mathrm{PH}$ within this Canadian province. While there were some 
technological issues identified by the participants, the vast majority of these barriers could be linked back to ineffective leadership and direction. Technological solutions might theoretically address issues such as poor data quality, but they can do little to 'fix' the mandates on the use of information systems and data sharing within the province, or varying interpretations of the legislation and obligations relating to HRs. This paper provided some evidence that the province was in alignment with the Gartner Hype Cycle, which stated blockchain technology was five to ten years away from mass adoption. This study suggested that these technologies were not well understood by PH informatics professionals. However, in order for blockchain to be successfully implemented in healthcare, it may not be necessary for each stakeholder to be au fait with the technical underpinnings of the solution; rather, the case for integrating blockchain will be made through empirical evidence of its benefits within the sector. Future research should build on this study by examining the views of other stakeholders in the province such as the other HRs and the provincial government, while also evaluating pilot projects that have introduced blockchain into $\mathrm{PH}$ in Canada and overseas.

While blockchain technology may be considered an 'immature' technology in healthcare, it already appears to be having an impact in other sectors in Canada. The Canadian government, specifically Canada Border Services Agency, is piloting a blockchain solution named 'TradeLens' for digital supply chain management for all shipments entering the country [42]. The National Research Council of Canada recently completed an experiment, a proof of concept use case of blockchain technology to publicly display contributions and grants in real time to support proactive disclosures to the public [43]. A 2018 study investigated the use of blockchain technology to link the existing EMRs across various health organisations via a unique identifier in China [44]. This Canadian province has an established unique health identifier for each citizen, and this information is held within the PCR database, under the control of the provincial government. To expedite the implementation of blockchain technology, this unique identifier could be leveraged by the province as a means to connect the various existing systems currently in use. This could potentially minimise the disruption to the front-end users of the clinical systems, while also providing a means to connect individual patient's health records across geographical boundaries supporting $\mathrm{PH}$ activities at every level.

\section{Conclusion}

A number of barriers currently exist which prevent the effective sharing of $\mathrm{PH}$ data in this Canadian province. These include technical issues, but overwhelmingly are of a human nature relating to interpretation of legislation, and a lack of effective management. In terms of the applicability of blockchain technology to counteract these barriers, efforts could be focussed on ensuring the existing systems are connected in a safe and secure manner. This allows for the existing systems to be built upon and integrated without the need to start from the beginning, causing disruption and confusion for the $\mathrm{PH}$ professionals using the systems. This research concludes that while blockchain technology shows promise for enhancing data sharing in healthcare, it is still many years away from being implemented in this Canadian province. More in-depth analysis is required in order to clearly understand how the technology could be applied, which blockchain format is appropriate and what benefits and risks it will bring. Future work should explore the perspectives of other stakeholders, such as the provincial government to fully understand the feasibility of using blockchain to share PH data in this context. Further research at a national level would also be beneficial to investigate whether similar barriers exist in other provinces and territories, with the goal of a pan-Canadian PH system, particularly important in the context of disease management. However, immediate efforts should focus on the human related barriers highlighted by the participants in this study.

\section{Declarations}

Conflict of interest The authors declare that they have no conflict of interest.

\section{References}

1. CDC, About CDC 24-7, Centers for Disease Control and Prevention. 2018. https://www.cdc.gov/about/default.htm Accessed 16 Apr 2018.

2. Mahon BE, Shea KM, Dougherty NN, Loughlin AM. Implications for registry-based vaccine effectiveness studies from an evaluation of an immunization registry: A cross-sectional study. BMC Public Health. 2008;8:160. https://doi.org/10.1186/1471-2458-8-160.

3. Papadouka V, Metroka A, Zucker JR. Using an Immunization Information System to Facilitate a Vaccine Recall in New York City, 2007. J Public Health Manag Pract : JPHMP. 2011;17:565-8. https://doi.org/10.1097/PHH.0b013e3182214746.

4. Heymann DL. The international response to the outbreak of SARS in 2003, Philosophical Transactions of the Royal Society of London. Series B: Biol Sci . 2004;359:1127-9. https://doi. org/10.1098/rstb.2004.1484.

5. Shearer FM, Moss R, McVernon J, Ross JV, McCaw JM, Infectious disease pandemic planning and response: Incorporating decision analysis, PLoS Med. 2020;17. https://doi.org/10.1371/journal. pmed.1003018.

6. Xu B, Kraemer MUG. Open access epidemiological data from the COVID-19 outbreak. Lancet Infect Dis. 2020. https://doi. org/10.1016/S1473-3099(20)30119-5.

7. van Panhuis WG, Paul P, Emerson C, Grefenstette J, Wilder R, Herbst AJ, Heymann D, Burke DS. A systematic review of barriers to data sharing in public health. BMC Public Health. 2014;14:1144. https://doi.org/10.1186/1471-2458-14-1144. 
8. Bellringer C, An Audit of the Panorama Public Health IT System I Auditor General of British Columbia, 2015. http://www.bcauditor. com/pubs/2015/audit-panorama-public-health-it-system Accessed 16 Apr 2018

9. Lee B, Martin T, Khan A, Fullerton K, Duck W, Kinley T, Stoutenburg S, Hall J, Crum M, Garcia MC, Iademarco MF, Richards CL. Modernizing Centers for Disease Control and Prevention Informatics Using Surveillance Data Platform Shared Services. Public Health Rep. 2018;133:130-5. https://doi.org/10.1177/0033354917751130.

10. Chan M, Kazatchkine M, Lob-Levyt J, Obaid T, Schweizer J, Sidibe M, Veneman A, Yamada T. Meeting the Demand for Results and Accountability: A Call for Action on Health Data from Eight Global Health Agencies. PLoS Med. 2010;7:e1000223. https://doi.org/10.1371/journal.pmed.1000223.

11. Walport M, Brest P. Sharing research data to improve public health. The Lancet. 2011;377:537-9. https://doi.org/10.1016/ S0140-6736(10)62234-9.

12. Stevens GA, Alkema L, Black RE, Boerma JT, Collins GS, Ezzati M, Grove JT, Hogan DR, Hogan MC, Horton R, Lawn JE, Marušić A, Mathers CD, Murray CJL, Rudan I, Salomon JA, Simpson PJ, Vos T, Welch V. Guidelines for Accurate and Transparent Health Estimates Reporting: the GATHER statement. The Lancet. 2016;388:e19-23. https://doi.org/10.1016/S01406736(16)30388-9.

13. Laine C, Goodman SN, Griswold ME, Sox HC. Reproducible Research: Moving toward Research the Public Can Really Trust. Ann Intern Med. 2007;146:450. https://doi.org/10.7326/00034819-146-6-200703200-00154.

14. Murray CJ. Towards good practice for health statistics: lessons from the Millennium Development Goal health indicators. The Lancet. 2007;369:862-73. https://doi.org/10.1016/S01406736(07)60415-2.

15. Abu-elezz I, Hassan A, Nazeemudeen A, Househ M, Abd-alrazaq A. The benefits and threats of blockchain technology in healthcare: A scoping review. Int J Med Informatics. 2020;142:104246. https://doi.org/10.1016/j.ijmedinf.2020.104246.

16. Antonopoulos A, Mastering Bitcoin: Programming the Open Blockchain, 2nd ed., O'Reilly. 2017.

17. Shah B, Shah N, Shakhla S, Sawant V. Remodeling the Healthcare Industry by employing Blockchain Technology, in. Int Conf Circuits Syst Digit Enterp Technol (ICCSDET). 2018;2018:1-5. https://doi.org/10.1109/ICCSDET.2018.8821113.

18. Gartner, Hype Cycle for Blockchain Business Shows Blockchain Will Have a Transformational Impact across Industries in Five to 10 Years, Gartner. (n.d.). 2019. https://www.gartner.com/en/ newsroom/press-releases/2019-09-12-gartner-2019-hype-cyclefor-blockchain-business-shows Accessed 2 Jul 2020.

19. Nakamoto S, Bitcoin: A Peer-to-Peer Electronic Cash System. 2008. https://bitcoin.org/en/bitcoin-paper Accessed 11 Apr 2018.

20. Kassab MH, DeFranco J, Malas T, Laplante P., Destefanis G, Neto VVG, Exploring Research in Blockchain for Healthcare and a Roadmap for the Future, IEEE Trans Emerg Top Comput. 2019;1-1. https://doi.org/10.1109/TETC.2019.2936881.

21. Engelhardt MA. Hitching Healthcare to the Chain: An Introduction to Blockchain Technology in the Healthcare Sector. Technol Innov Manag Rev. 2017;7:22-34.

22. Agbo CC, Mahmoud QH, Eklund JM, Blockchain Technology in Healthcare: A Systematic Review, Healthcare (Basel). 2019;7. https://doi.org/10.3390/healthcare7020056.

23. Hölbl M, Kompara M, Kamišalić A, Zlatolas LN. A Systematic Review of the Use of Blockchain in Healthcare. Symmetry. 2018;10:470. https://doi.org/10.3390/sym10100470.

24. Angraal S, Krumholz H, Schulz WL, Blockchain Technology: Applications in Health Care, Circulation: Cardiovascular Quality and Outcomes. 2017;10:e003800. https://doi.org/10.1161/ CIRCOUTCOMES.117.003800.
25. Mettler M, Blockchain technology in healthcare: The revolution starts here, in: 2016 IEEE 18th International Conference on E-Health Networking, Applications and Services (Healthcom), 2016;1-3. https://doi.org/10.1109/HealthCom.2016.7749510.

26. Heston T, A case study in blockchain health care innovation, International Journal of Current Research. 2017;9:60587-60588. https:// doi.org/10.22541/au.151060471.10755953.

27. Cohen JK, 15 best papers on blockchain in health IT, as chosen by HHS. 2017. https://www.beckershospitalreview.com/healthcareinformation-technology/15-best-papers-on-blockchain-in-healthit-as-chosen-by-hhs.html Accessed 15 Apr 2018.

28. Orcutt M, Why the CDC thinks blockchain can save lives, MIT Technology Review. 2017. https://www.technologyreview. com/s/608959/why-the-cdc-wants-in-on-blockchain/ Accessed 31 Mar 2018.

29. Clim A, Zota RD, Constantinescu R. Data exchanges based on blockchain in m-Health applications. Procedia Computer Science. 2019;160:281-8. https://doi.org/10.1016/j.procs.2019.11.088.

30. JMIR - Towards a Stakeholder-Oriented Blockchain-Based Architecture for Electronic Health Records: Design Science Research Study I Beinke I Journal of Medical Internet Research, (n.d.). https:// www.jmir.org/2019/10/e13585 Accessed 6 Feb 2021.

31. Chen H, Huang X. Will Blockchain Technology Transform Healthcare and Biomedical Sciences? EC Pharmacol Toxicol. 2018;6:910-1.

32. Health Canada, Canada's health care system, Aem. 2016. https:// www.canada.ca/en/health-canada/services/canada-health-caresystem.html Accessed 14 May 2020.

33. Phillips B. Understanding experience through Gadamerian hermeneutics: an interview with Brian Phillips. Interview by Pamela Wood \& Lynne Giddings, Nurs Prax N Z. 2005;21:3-14.

34. Creswell JW, Qualitative inquiry \& research design: choosing among five approaches., Fourth edition, International student edition / John W. Creswell, Cheryl N. Poth., SAGE, Los Angeles. 2018.

35. Liamputtong P. Qualitative research methods. 2nd ed. Vic.; Oxford: Oxford University Press, South Melbourne; 2005.

36. Errasti-Ibarrondo B, Jordán JA, Díez-Del-Corral MP, Arantzamendi M. Conducting phenomenological research: Rationalizing the methods and rigour of the phenomenology of practice. J Adv Nurs. 2018;74:1723-34. https://doi.org/10.1111/jan.13569.

37. Gadamer HG, Truth and method, 2nd rev. ed., translation revised by Joel Weinsheimer < span class="searchword" $>$ and $</$ span $>$ Donald G. Marshall., Sheed \& Ward, London, 1989.

38. Tracy SJ. Qualitative Research Methods: Collecting Evidence. Communicating Impact, Wiley: Crafting Analysis; 2012.

39. Fontana A, Frey JH, The Interview: From Structured Questions to Negotiated Text, in: Handbook in Qualitative Research, 2nd Ed., Thousand Oaks, CA, London, New Delhi: Sage Publications, 2000:645-672.

40. Clarke V, Braun V. Using thematic analysis in counselling and psychotherapy research: A critical reflection. Couns Psychother Res. 2018;18:107-10. https://doi.org/10.1002/capr.12165.

41. Heidegger M. Being and time. Oxford: Blackwell; 1990.

42. C.B.S.A. Government of Canada, The CBSA Pilots Blockchain Technology. 2018. https://www.cbsa-asfc.gc.ca/new-neuf/articles/ blockchain-chaine-blocs-eng.html Accessed 23 Aug 2019.

43. Blockchain publishing prototype - NRC-IRAP, (n.d.). https://nrccnrc.explorecatena.com/en Accessed 23 Aug 2019.

44. Cheng EC, Le Y, Zhou J, Lu Y. Healthcare services across China - on implementing an extensible universally unique patient identifier system. Int J Healthc Manag. 2018;11:210-6. https://doi. org/10.1080/20479700.2017.1398388. 